\section{Additive and subtractive color mixture in color transparency}

\section{JACOB BECK \\ University of Oregon, Eugene, Oregon 97403}

Perceptual transparency refers to the phenomenon of perceiving one color through another. Heider (1933) hypothesized that the overlapping planes of color into which a stimulus color might be scissioned were those which, when additively mixed together, yield the stimulus color. Thus, if a gray stimulus color gives rise to the perception of a transparent overlying blue film, then the underlying opaque color will be seen as yellow. Metelli (1974a) advanced the theory of transparency by deriving quantitative formulas to predict color transparency with achromatic colors. He assumed that perceptual scission in transparency quantitatively followed Talbot's law. Consider the case where perceptual transparency is obtained by rotating an episcotister (a sheet with open sectors) in front of two opaque surfaces, $A$ and $B$, located behind an episcotister, $E$ (Figure 1). Let $a=$ the reflectance of surface $A ; b=$ the reflectance of surface $B ; t=$ the reflectance of the episcotister $E ; p=$ the stimulus color resulting from the episcotister rotating in front of surface $\mathrm{A} ; \mathrm{q}=$ the stimulus color resulting from the episcotister rotating in front of surface B; $\alpha=$ the proportion of which the stimulus colors $\mathrm{p}$ and $\mathrm{q}$ are made up of the reflectances $a$ and $b$ (that is, the open sectors of the episcotister), and $1-\alpha=$ the proportion of which the stimulus colors, $\mathrm{p}$ and $\mathrm{q}$, are made up of the reflectance of the episcotister, $E$ (that is, the episcotister blades). According to Talbot's law, the stimulus colors $\mathrm{p}$ and $q$ are equal to:

$$
\begin{gathered}
\mathrm{p}=\alpha \mathrm{a}+(1-\alpha) \mathrm{t} \\
\mathrm{q}=\alpha \mathrm{b}+(1-\alpha) \mathrm{t}
\end{gathered}
$$

Solving Equations 1 and 2 for $\alpha$ and $\mathrm{t}$ yields:

$$
\alpha=\mathrm{p}-\mathrm{q} / \mathrm{a}-\mathrm{b}^{1}
$$

and

$$
t=a q-b p /(a+q)-(b+p) .
$$

The values $\alpha$ and $1-\alpha$ are the proportions into which the stimulus colors $\mathrm{p}$ and $\mathrm{q}$ split into the reflectances $a$ and $t$, and $b$ and $t$. The smaller the value of $\alpha$, the more of the color which goes into the episcotister plane and the less its transparency. If $\alpha=.5$, then the blades of the episcotister are half open and the color is divided evenly between the episcotister and the background surfaces $(\mathrm{p}=.5 \mathrm{a}$ $+.5 \mathrm{t}$ and $\mathrm{q}=.5 \mathrm{~b}+.5 \mathrm{t})$. One can then see a semitransparent circle of uniform reflectance in front of a background $\mathrm{A}$ of reflectance $\mathrm{a}$ and of a background $B$ of reflectance $b$. Equations 3 and 4 set conditions for the perception of transparency. Since $\alpha$ is restricted to values between 0 and 1 , transparency is impossible if the difference between reflectances $p-q$ is greater than that between reflectances $a-b$. Transparency is also impossible if the direction in the change of reflectances is not the same for $p-q$ and for $a-b$, i.e., if $p$ has a higher reflectance than $q$ and $b$ has a higher reflectance than $a$. Metelli (1974a, 1974b) presents examples where the conditions set by Equations 3 and 4 are satisfied and transparency may be obtained, and where the conditions are not satisfied and transparency does not occur.

Beck $(1972,1975)$ proposed the hypothesis that color transparency might also occur in terms of subtractive color mixture. Subtractive color mixture takes place whenever the light is affected by selectively absorbing materials. Quantitatively, subtractive color mixture is determined by the successive application of the percentage transmission of each absorbing material to the energy distribution of the light source. Common occurrences are when paints of different

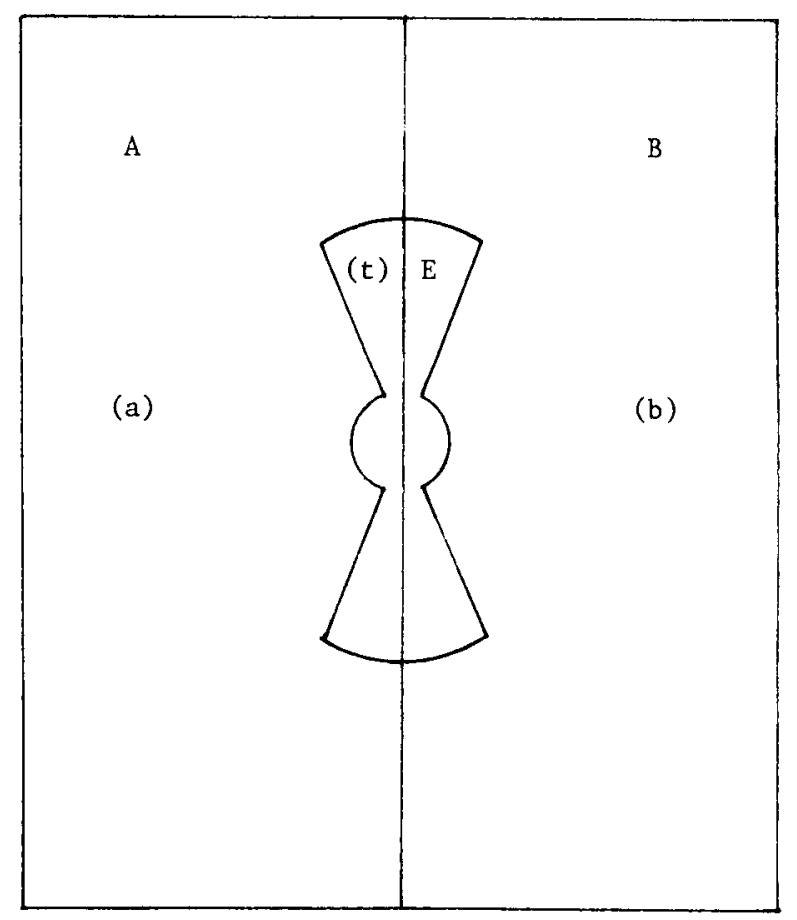

Figure 1. Episcotister arrangement: $\mathbf{A}=\operatorname{surface} \mathbf{A}$, (a) $=$ reflectance of surface $A, B=$ surface $B,(b)=$ reflectance of surface $B, E=$ episcotister, and $(t)=$ reflectance of episcotister. 
colors are mixed or when a person looks at a surface through a pane of colored glass. The purpose of this note is to show that, for achromatic colors, a model based on subtractive color mixture yields the same equations as a model based on additive color mixture.

Let $\mathrm{F}$ be an achromatic filter through which we look at surfaces $\mathrm{A}$ and $\mathrm{B}$. Let $\mathrm{a}=$ the reflectance of surface $A ; b=$ the reflectance of surface $B$; $\mathrm{m}=$ the transmittance of the filter; $\mathrm{r}=$ the reflectance of the filter; $s=$ the absorbtion of the filter; $p=$ the stimulus color resulting from the filter in front of the surface $A ; q=$ the stimulus color resulting from the filter in front of the surface $B$. A filter reduces the amount of light transmitted through either reflection or absorbtion of the incident light. The light transmitted, plus the light reflected plus the light absorbed is equal to the light incident on the filter; that is, $m+r+s=1$. The light reaching the eyes is equal to the amount of light transmitted by the filter plus the amount of light reflected from the front surface of the filter. The amount of light transmitted from surfaces A and B is equal to $\mathrm{ma}$ and $\mathrm{mb}$. The proportion of incident light reflected by the filter is $r$. The stimulus colors $p$ and $q$ are equal to:

$$
\begin{aligned}
& \mathrm{p}=\mathrm{ma}+\mathrm{r} \\
& \mathrm{q}=\mathrm{mb}+\mathrm{r} .
\end{aligned}
$$

A one-to-one correspondence will be shown to hold between the formulas based on the episcotister model (Equations 1 and 2) and the formulas based on a filter model (Equations 5 and 6). This will be done in two steps. First, every achromatic filter will be shown to be reducible to a canonical filter consisting of clear and opaque points, and second, a one-to-one correspondence will be established between the formulas based on a canonical filter and the formulas based on an episcotister model.

In the case of an achromatic filter, there is a minimal area for which its visual effect is dependent upon the total amount of light transmitted and not upon the distribution of light within this area. Such an area, for example, is a retinal receptor or a receptive field which integrates all the stimulation falling upon it. The canonical filter is constructed in the following way. The filter is divided into minimal areas. Within each of these minimal areas, the filter is constructed out of clear points that transmit all the incident light and opaque points that transmit no light. The opaque points of different canonical filters may differ in the amounts of incident light that they reflect and absorb. For a given filter, let $c=$ the proportion of points which are clear and transmit all the incident light; $T_{c}=$ the transmittance of the clear points and equals
$1 ; 0=$ the proportion of points which are opaque and transmit no light; $R_{o}=$ the reflectances of the opaque points. The stimulus colors $p$ and $q$ resulting from surfaces $A$ and $B$, respectively, are equal to:

$$
p=c a+o R_{o}
$$

and

$$
q=c b+o R_{0} \text {. }
$$

Since the amount transmitted and reflected within each minimal area is the same, Equations 7 and 8 apply to the whole filter. Since $T_{c}$, by assumption, is 1 , c determines the amount of light transmitted and is equal in Equations 7 and 8 to $\mathrm{m}$ in Equations 5 and 6 . The amount of light reflected by the canonical filter is: $r=0 \times R_{0}$. This defines a correspondence between a conventional filter and a canonical filter.

A one-to-one correspondence will now be established between a canonical filter and the Metelli equations based on an episcotister model. Since $c+o=1$ (the entire filter is composed of the clear points plus the dense points), Equations 7 and 8 can be written as:

$$
p=c a+(1-c) R_{o}
$$

and

$$
q=c b+(1-c) R_{0} .
$$

Light coming to the eyes is composed of two parts; $\mathrm{ca}$ and $\mathrm{cb}$ represent the light coming from the surfaces $A$ and $B$ and $(1-c) R_{o}$ represents the light reflected from the filter. Solving for $c$ and $R_{o}$ yields:

$$
c=p-q / a-b
$$

and

$$
R_{o}=a q-b p /(a+q)-(b+p) .
$$

From Equations 3 and $4, c=\alpha$ and $R_{o}=t$. Thus, there is an isomorphic correspondence between the equations based on the episcotister model and equations based on a filter model. It follows that any experimental outcomes predicted by the episcotister model is predicted by the filter model, and one cannot distinguish experimentally between these two models.

Metelli and his colleagues have recently begun to deal with the question of the perception of transparency with hue. Da Pos $(1976 \mathrm{a}, 1976 \mathrm{~b})$ has presented an analysis of color transparency based on an 
additive color mixture. Beck $(1972,1975)$ hypothesized that hue transparency occurs in terms of subtractive color mixture. Since looking at a surface through a chromatic filter changes the intensities of the wavelengths transmitted nonuniformly, a chromatic filter cannot be reduced to a filter consisting of clear and opaque points. Thus, hue transparency allows us to distinguish between additive and subtractive color mixture. For example, yellow and blue produce a gray in the case of additive color mixture and a green in the case of subtractive color mixture. Metelli (1974a) has shown that a compelling perception of transparency may be produced by juxtaposing opaque papers of differing reflectance. I have been able to demonstrate hue transparency in which a green color is seen as a yellow color through a blue, or vice versa, using both paints and colored papers employing the mosaic method developed by Metelli.

The fact that color transparency can occur in accordance with subtractive as well as additive color mixture suggests that colors as they occur in everyday experience may facilitate the phenomenon of transparency. However, this does not mean that color transparency is entirely arbitrary and that any combination of hues can produce the phenomenon of transparency. In theory, of course, it is possible to design dyes so that any given two colors can be made to yield a third color with subtractive color mixture. The hypothesis I would like to propose is that the perception of transparency depends on the visual system encoding a stimulus color as a given color plus a deviation due to the presence of an overlapping color film, i.e., a prototypical color plus a correction (Beck, 1972). The encoding of a stimulus color as a prototypical color plus a correction depends on both figural and color conditions. Heider (1933) found that the impression of transparency depended on figural factors favoring the perception of two superimposed surfaces. The color conditions favoring transparency with chromatic colors are at present unclear. Stimulus support, however, may be required for perceiving a stimulus color as one color through another. Two types of stimulus support are possible with both additive and subtractive color mixture. First, the mixture color may not be pure and may appear to contain an admixture of one of the reference colors. Secondly, stimulus support may be provided in terms of flecks of hue, contrast colors at edges, or highlights from the light source which are the colors of the reference colors. Discrete stimulus inhomogeneities are able to produce the perception of a continuous surface (Gibson, Purdy, \& Lawrence, 1955). For example, highlights at discrete points produce the perception of glossiness over the entire surface (Beck, 1972). It is possible that color transparency is also facilitated by similar completion processes and that discrete inhomogeneities in a stimulus color can produce the perception of a continuous overlying film of color.

\section{REFERENCES}

BECK, J. Surface color perception. Ithaca: Cornell University Press, 1972.

BECK, J. Surface color. Scientific American, 1975, 233(2), 62-75.

DA Pos, O. Perceptual transparency: Additive or subtractive chromatic mixture? Atti e Memorie dell'Academia Patavina di Scienza-Parte III, 1976, 185-193. (a)

DA Pos, O. Contributo tearico-sperimentale alle percezione della trasparanza equilibrata con colore. Atti dell'Istituto Veneto di Scienza, 1976, 701-722. (b)

Heider, G. M. New studies in transparency, form, and colour. Psychologische Forschung, 1933, 17, 13-56.

Gibson, J. J., Purdy, J., \& Lawrence, L. A method for controlling stimulation for the study of space perception: The optical tunnel. Journal of Experimental Psychology, 1955, 50, 1-14.

METELLI, F. Achromatic color conditions in the perception of transparency. In R. B. MacLeod \& H. L. Pick (Eds.), Perception: Essays in honor of $J$. J. Gibson. Ithaca: Cornell University Press, 1974. Pp. 96-116. (a)

Metelli, F. The perception of transparency. Scientific American, 1974, 230(4), 90-98. (b)

\section{NOTE}

1. I followed Metelli and expressed the transparency index, $\alpha_{3}$ in Equation 3 in terms of the variable of reflectance. It should be pointed out that actual judgments of transparency more than likely will not vary linearly with reflectance differences. Rather, judgments of transparency may be expected to vary linearly with lightness differences. Thus, to predict actual judgments of transparency, one must introduce into Equation 3 a psychophysical function, such as the Munsell value scale, which describes how the magnitude of subjective lightness differences vary as a function of reflectance differences.

(Received and accepted for publication January 20, 1978.) 